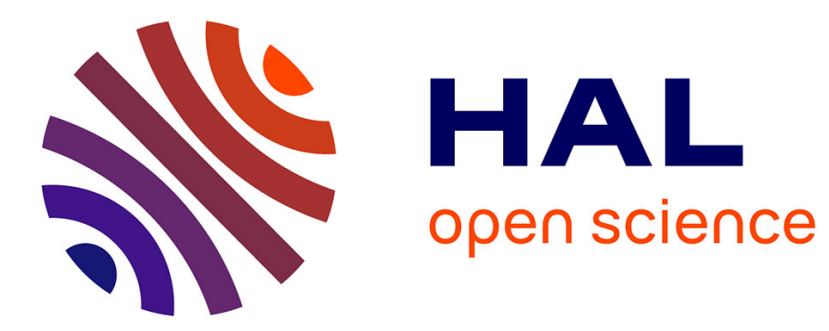

\title{
Métropoles émergentes et alimentation : une lecture croisée
}

Gwenn Pulliat

\section{To cite this version:}

Gwenn Pulliat. Métropoles émergentes et alimentation : une lecture croisée. L'Information géographique, 2017, 81 (3), 10.3917/lig.813.0054 . hal-01921818

\section{HAL Id: hal-01921818 https://hal.science/hal-01921818}

Submitted on 14 Nov 2018

HAL is a multi-disciplinary open access archive for the deposit and dissemination of scientific research documents, whether they are published or not. The documents may come from teaching and research institutions in France or abroad, or from public or private research centers.
L'archive ouverte pluridisciplinaire HAL, est destinée au dépôt et à la diffusion de documents scientifiques de niveau recherche, publiés ou non, émanant des établissements d'enseignement et de recherche français ou étrangers, des laboratoires publics ou privés. 


\title{
Métropoles émergentes et alimentation : une lecture croisée
}

\author{
Par Gwenn Pulliat
}

Gwenn Pulliat, Chercheuse associée, IRASEC (Institut de recherche sur l'Asie du Sud-Est contemporaine), chercheuse associée post-doctorante, University of Toronto -gwenn.pulliat@gmail.com

\section{- Introduction}

En janvier 2017, la revue annuelle du club Déméter, qui regroupe des dirigeants du monde agricole français, consacre un long dossier à l'alimentation de la Chine et ses 1,4 milliard d'habitants. La croissance urbaine (et son corollaire, l'urbanisation des terres agricoles) est citée comme un défi majeur pour la sécurité alimentaire du pays ; le pays doit, de plus en plus, importer des denrées alimentaires. Pour répondre à ces enjeux, les autorités publiques investissent fortement dans le domaine agricole et développent une stratégie d'acquisition de terres à l'international. À une échelle large, la situation chinoise rend compte de la manière dont s'articulent les différentes filières d'approvisionnement pour répondre à des besoins croissants .

L'alimentation se fonde sur un système fortement spatialisé (Ericksen, 2008 ; Rastoin et Ghersi, 2010 ; Sobal, Kettel Khan et Bisogni, 1998). Les espaces de production et les espaces de consommation, largement découplés, interagissent et sont reliés par des dispositifs d'approvisionnement qui assurent la distribution des denrées jusqu' aux consommateurs. Fait spatial donc, mais aussi fait social : les pratiques alimentaires diffèrent selon le contexte culturel, le cadre de vie, le niveau de richesse, pour ne citer que quelques-uns des facteurs influençant les habitudes des individus. Aussi, dans un monde qui est désormais majoritairement urbain, une attention croissante est-elle portée aux enjeux de l'alimentation des villes (Bricas et Seck, 2004 ; Pothukuchi et Kaufman, 1999).

Caractérisée par une concentration des hommes et des activités dans un espace densément occupé, la ville représente un objet d'étude particulier dans le champ alimentaire : la pression foncière conduit à repousser les zones de production de plus en plus loin, alors que la croissance démographique, mais aussi l'augmentation du niveau de vie conduisent à accroître la demande. L'enjeu que représente la sécurité alimentaire urbaine a été rappelé par les mouvements sociaux consécutifs à la crise de 2008. Qualifiés, par les médias, 
d'émeutes de la faim, ils ont été menés par des groupes sociaux précarisés et particulièrement sensibles aux fluctuations des prix (Cohen et Garrett, 2010 ; Dessus, Herrera et De Hoyos, 2008 ; Janin, 2009). Aussi, en raison des dynamiques de croissance et des enjeux politiques associés, les études sur la sécurité alimentaire se sont largement emparées du champ urbain, enrichissant son cadre d'analyse : la question de la nutrition, notamment, est désormais incontournable (Carletto, Zezza et Banerjee, 2013 ; Coates, Swindale et Bilinsky, 2007 ; Maxwell et al., 2000).

Dans ce contexte, les régions urbaines des Suds constituent un enjeu particulièrement saillant, parce que le rythme de leur développement, tant démographique et spatial qu'économique, impose des transformations à la fois rapides et profondes de leur système alimentaire.

Suivant le cadre conceptuel proposé par Ericksen (2008), le système alimentaire, dont l'objectif premier est de garantir la sécurité, assure trois fonctions principales : la disponibilité de l'alimentation, son accessibilité et enfin son utilisation. Aussi, considérer les villes du point de vue de leur système alimentaire donne à voir une image de leur évolution : évolution des rapports entre la ville et son hinterland, transformation des canaux d'approvisionnement qui traduisent les mutations des usages de la ville, modifications des habitudes de consommation accompagnées d'une stratification sociale croissante... L'alimentation constitue alors une grille de lecture pertinente des recompositions des régions urbaines des Suds.

L'article se fonde sur une étude des pratiques alimentaires menées à Hanoï (Vietnam) en 2011-2012 ${ }^{1}$, complétée par les premiers résultats d'une recherche portant sur les espaces agricoles urbains et périurbains menée à Bangkok (Thaillande) à l'automne $2016^{2}$.

- Centré sur la construction de la sécurité alimentaire à l'échelle individuelle, le premier travail de terrain s'est appuyé sur la réalisation de 100 récits de vie menés auprès de résidents de trois quartiers de la ville, l'un central (Phuc Tan) et les deux autres (Yen So et An Khanh) situés à l'interface entre le pôle urbain central et l'espace périurbain de la région. Ces deux quartiers périphériques connaissent un processus d'étalement urbain rapide.

- Le deuxième travail porte sur les dynamiques des espaces agricoles au sein de la ville de Bangkok et de ses périphéries : il est appuyé sur une vingtaine

1. Enquête de terrain menée dans le cadre des travaux de thèse de l'auteur, menés à l'Université Paris Ouest.

2. Enquête de terrain menée dans le cadre de la bourse postdoctorale de terrain et de publication attribuée par l'Institut de Recherche sur l'Asie du Sud Est Contemporaine (IRASEC) en 2016. 
d'entretiens menés auprès de producteurs, de personnes engagées dans différents groupes de défense de l'environnement, de responsables de programmes politiques environnementaux et de chercheurs thaïlandais. Les espaces agricoles étudiés sont principalement situés dans la province de Nonthaburi à l'ouest et dans le quartier de Minburi à l'est.

\section{- Flux alimentaires et système urbain : une lecture des interactions entre la ville et son espace environnant}

\section{Des relations d'interdépendance entre la ville et son hinterland}

Pour assurer la disponibilité d'une alimentation satisfaisant les besoins de leur population, les villes recourent nécessairement à un espace d'approvisionnement plus ou moins vaste : en ce sens, elles « externalisent les conditions matérielles de leur subsistance » (Bognon, 2015). Les relations entre les villes et les espaces qui les approvisionnent sont donc primordiales. La production agricole au sein des régions urbaines demeurent limitée, de sorte que l'accès à l'alimentation se fonde majoritairement sur le recours au marché (Sen, 1981).

Aussi, à l'échelle métropolitaine, l'étude des flux alimentaires met-elle en évidence les relations d'interdépendance entre la ville et son hinterland. Hanoï se situe au cœur du delta du Fleuve Rouge, une des deux régions majeures pour la production agricole au Vietnam avec le delta du Mékong au sud. Si la taille très réduite des exploitations implique qu'une part importante des produits soient autoconsommés ou vendus localement (Dao et Fanchette, 2008), le marché urbain hanoïen constitue néanmoins un débouché pour les productions du delta. C'est particulièrement vrai pour les produits périssables, dont les légumes-feuilles, denrées majeures dans le régime alimentaire des Vietnamiens. Sautier et al. (2012), qui ont mené une étude exploratoire, en 2011, sur des marchés de gros à Hanoï, ont montré que, si le bassin d'approvisionnement s'élargit vers les provinces limitrophes, $75 \%$ des liserons d'eau vendus au moment de leur étude avaient été produits dans la province. Pour ces produits frais périssables, les flux courts, de petits volumes et de haute fréquence, restent la norme.

À Bangkok, en première analyse, les espaces agricoles de la région métropolitaine paraissent globalement peu spécifiques, et très similaires aux espaces agricoles ruraux des plaines du pays - c'est-à-dire que les productions approvisionnent certes le marché urbain, mais aussi largement le reste du pays et le marché international (Poupon, 2011). Pour autant, une part de l'approvisionnement des ménages est assurée par les marchés informels («meeting 
Fig. 1 : Production de légumes dans la frange urbaine de Hanoï.

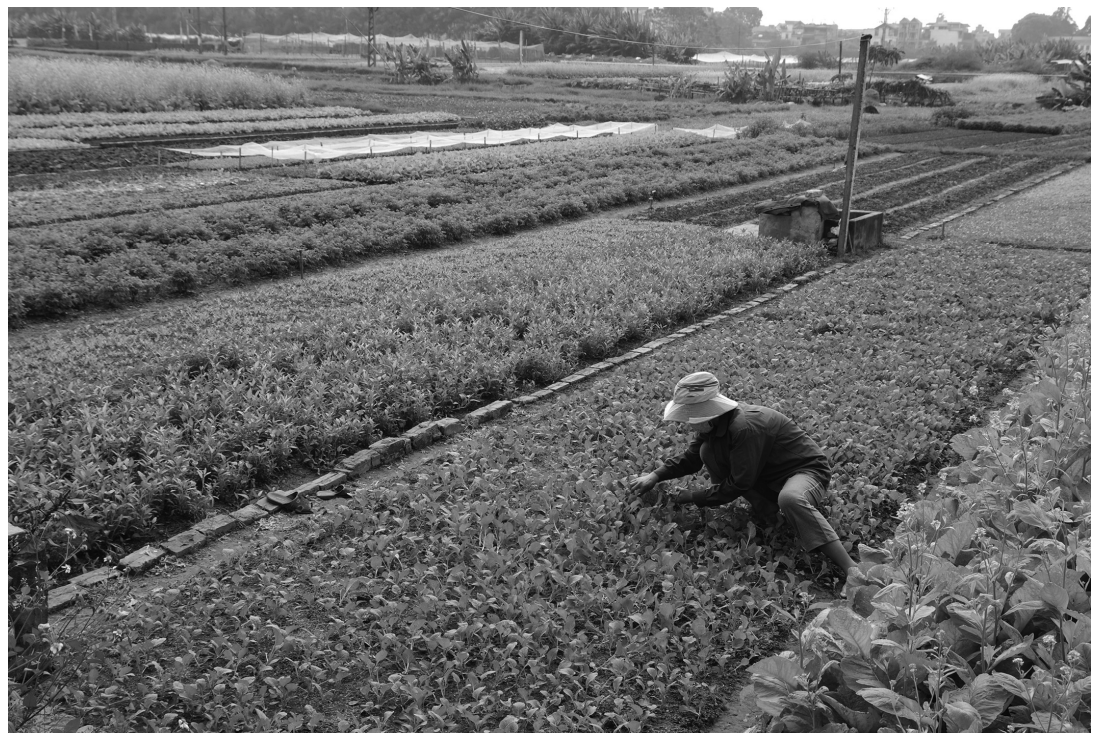

Photo de I'auteur, 2011.

markets ») : il s'agit là de circuits courts (avec au maximum un intermédiaire entre le producteur et l'acheteur) représentant un débouché pour les denrées provenant des alentours de la ville (jusqu'à deux heures de route environ). Des données supplémentaires seraient nécessaires pour évaluer la part de cette filière pour les producteurs autant que dans l'approvisionnement des consommateurs, mais ce marché informel rend compte du lien spécifique persistant entre hinterland agricole et marché urbain.

En outre, le marché urbain est surtout un moteur de diversification des productions : des denrées à forte valeur ajoutée, notamment les fruits, la volaille, le porc, les produits laitiers, connaissent un développement rapide, au détriment de cultures moins compétitives telles que celle du riz. Ainsi, par exemple, entre 2000 et 2007 les surfaces en riz de la province de Hanoï (dans ses anciennes limites) ont été réduites de $20 \%$, cependant que le nombre de porcs a crû de 35 \% (Sautier et al., 2012). L'élevage laitier s'est également développé, notamment dans le district de $\mathrm{Ba} \mathrm{Vi} \mathrm{(ouest} \mathrm{de} \mathrm{la} \mathrm{région}$ de Hanoï) : si son implantation remonte à l'époque coloniale, son essor est lié aux mutations économiques que connaît le Vietnam (Duteurtre et Pannier, 2016), et la proximité du marché urbain en est un accélérateur. C'est là un processus très classique qui traduit l'influence du développement urbain sur l'organisation spatiale de son hinterland : la pression foncière qui accroît le coût des terres agricoles, l'amélioration des transports qui favorise l'accès au marché urbain, l'augmentation de la population et de ses revenus en sont les 
Fig. 2 : Vente de fruits dans un pick-up : un exemple de filière courte informelle à Bangkok.

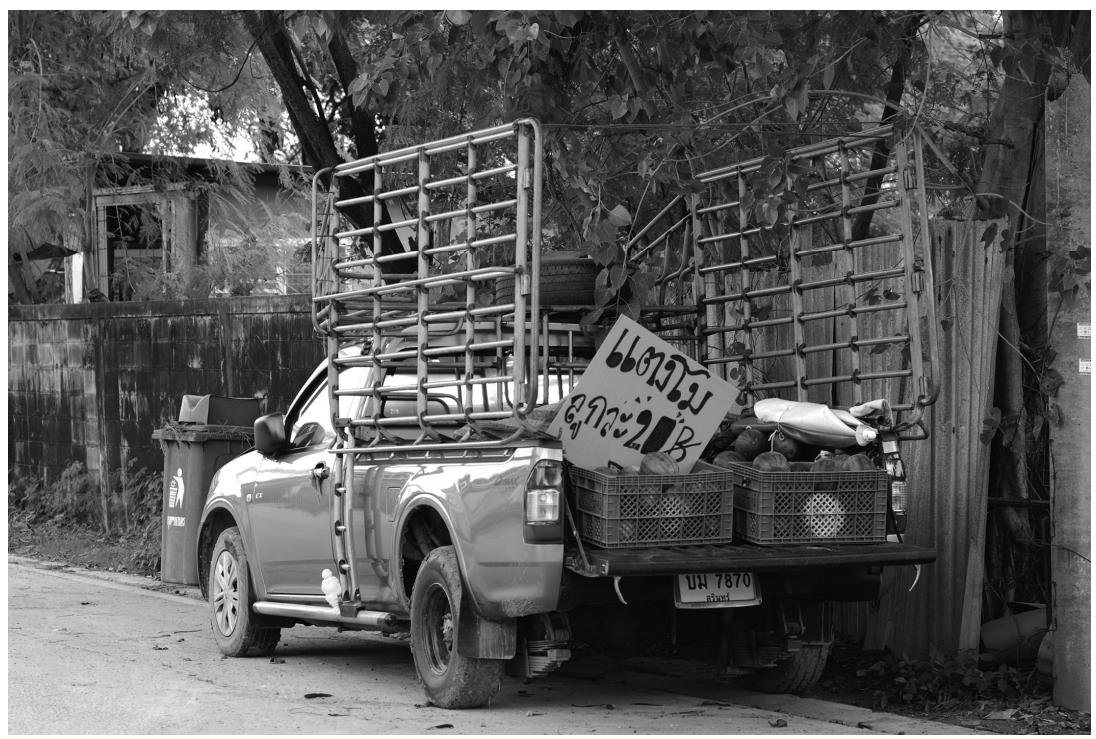

Photo de l'auteur, 2016.

facteurs principaux. Ainsi, l'évolution des productions agricoles rend compte d'une intégration croissante des espaces environnants au système urbain.

\section{Une intégration croissante des espaces métropolitains à la globalisation}

À une échelle plus large, l'évolution des sources d'approvisionnement de la capitale traduit elle aussi une intégration régionale et mondiale croissante. Au Vietnam, l'ouverture économique entamée avec le Doi Moi à la fin des années 1980, symbolisée notamment par l'adhésion du pays à l'Association des Nations d'Asie du Sud Est (ASEAN) en 1997 puis à l'Organisation Mondiale du Commerce (OMC) dix ans plus tard, lui a permis de devenir un acteur important sur plusieurs marchés alimentaires mondiaux. Le Vietnam est devenu un exportateur majeur pour certains produits bruts, notamment le riz dont il assure près de $20 \%$ de l'offre sur le marché mondial selon les données de la FAO. Les importations, quant à elles, répondent à la diversification des consommations alimentaires : blé, huile de palme, produits laitiers, produits transformés, mais aussi des produits destinés à l'alimentation animale. Aussi observe-t-on, à Hanoï, une diversification de l'origine des flux alimentaires. Pour les produits frais, le marché hanoïen constitue un débouché pour les productions du sud du pays, mais également de la Chine voisine, d'autant 
que, dans le cadre de la coopération entre la Chine et l'ASEAN, les barrières douanières ont été fortement abaissées en 2010. De surcroit, des flux de contrebande drainent les marchés de la capitale ; si leur mesure est périlleuse, leur existence est avérée. Les produits agro-industriels, quant à eux, ont une origine souvent plus lointaine : Chine, Corée du Sud, Japon, Europe, ÉtatsUnis...

Ainsi, l'étude des flux alimentaires de Hanoï témoigne d'un double processus d'intégration : celle de l'hinterland au système urbain, et celle de la métropole dans un ensemble supra-régional et mondial élargi. La métropolisation en cours s'accompagne donc d'une forme d'insertion dans la globalisation, avec des échanges à l'échelle mondiale et le développement de pratiques alimentaires marquées par une certaine « occidentalisation» (Pingali, 2007).

\section{- Les pratiques alimentaires des citadins : une lecture des changements sociaux}

\section{Développement urbain et mutations de la distribution alimentaire}

L'insertion dans un processus de globalisation se lit d'abord dans l'évolution des lieux d'achat. Le symbole fort de ce processus est le développement des supermarchés. Inexistants jusqu'en 1990, ils ont connu une croissance rapide depuis : Hanoï en dénombrait 32 en 2000 (Moustier et Dao, 2006), et 110 à la fin de l'année 2011 (Courrier du Vietnam, 2012). Ils sont notamment associés au déploiement de centres commerciaux, de malls souvent installés dans des tours qui poussent rapidement un peu partout dans la ville-centre, répondant à la volonté du Comité Populaire de Hanoï de promouvoir l'image d'une capitale émergente et attractive.

S'il s'agit souvent de surfaces relativement petites (et qui, de surcroît, n'ont pas toujours une vocation alimentaire), les supermarchés sont associés par les consommateurs à une image de qualité et de sûreté alimentaire (Figuié et al., 2004) - notamment parce qu'ils constituent un lieu de vente privilégié pour les « légumes sûrs », cultivés avec moins de produits phytosanitaires que les productions traditionnelles.

Toutefois, le déploiement des grandes surfaces est surtout lié à la commercialisation de produits agro-industriels, souvent importés (confiseries, pâtes et nouilles instantanées, produits pour bébés, plats préparés congelés par exemple), ainsi que des produits manufacturés d'utilisation courante : produits d'entretien, produits de beauté... Les produits frais y sont relativement peu représentés et les hypermarchés généralistes demeurent, à l'heure 
actuelle, peu nombreux. Il y a ainsi une certaine spécialisation des lieux de commercialisation selon les types de produits.

Parallèlement donc, le Comité Populaire de Hanoï entend réformer les espaces de vente des produits frais. Il mène ainsi une politique de rénovation des marchés, fondée, d'une part, sur une interdiction de certains marchés informels installés dans la rue et, d'autre part, sur la construction de bâtiments associés à des infrastructures améliorées (zones réfrigérées, évacuation des eaux usées, gestion des déchets, parkings) (Geertman, 2011). Il s'agit d'améliorer les conditions d'hygiène mais aussi de promouvoir l'image d'une ville propre et salubre : la vente de rue sied mal à l'image de la ville émergente (Anjaria, 2006 ; Monnet, 2006).

Il n'en demeure pas moins que cette forme d'approvisionnement dans la rue reste ancrée dans les habitudes des Hanoïens, surtout parce qu'elle a pour attribut d'être locale (Wertheim-Heck, Vellema et Spaargaren, 2015). Cette proximité assure le tissage de relations de confiance entre vendeurs et acheteurs : ces derniers peuvent ainsi obtenir les meilleurs prix et réaliser, quand nécessaire, des achats à crédit (Moustier et Dao, 2006). Enfin, les consommateurs estiment alors avoir une meilleure connaissance de l'origine des produits et transposent aux denrées la confiance qu'ils font au vendeur.

\section{Fig. 3 : Vente de rue fixe et ambulante à Hanoï : un mode d'approvisionnement qui persiste.}

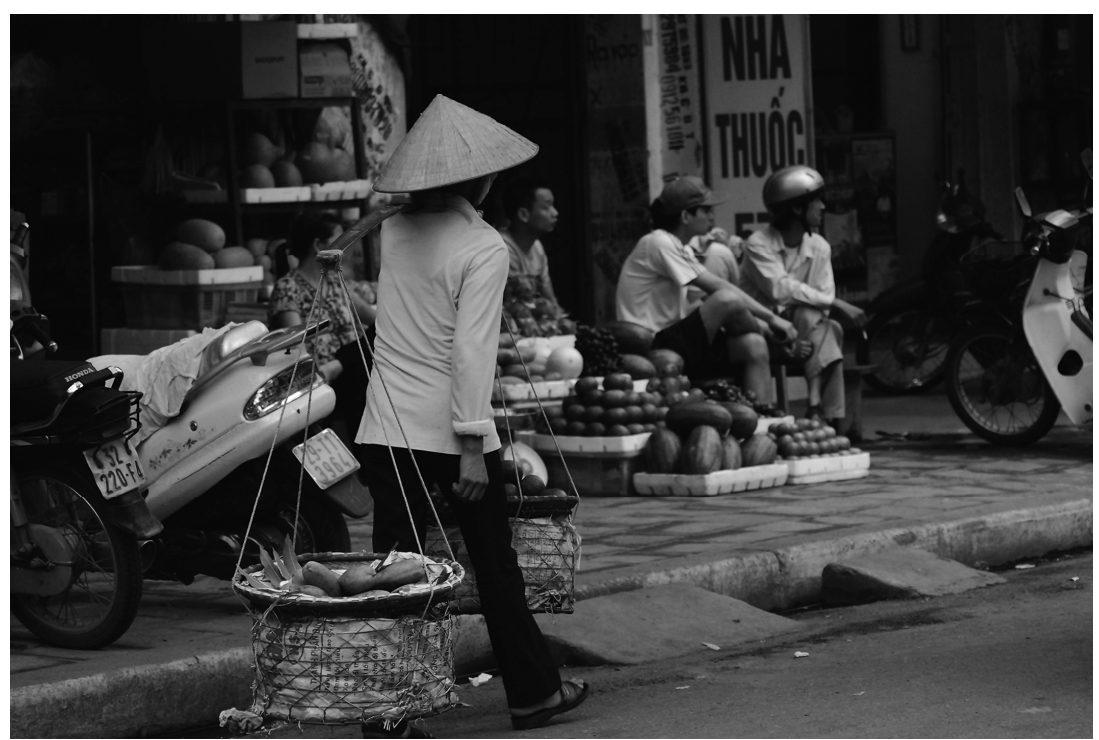

Photo de l'auteur, 2009. 
Aussi, l'observation des pratiques d'approvisionnement et de leur évolution met en évidence un processus de différenciation sociale croissante. Les supermarchés et les nouveaux marchés modernisés, plus onéreux que la vente de rue, sont surtout fréquentés par une population urbaine plutôt jeune et favorisée, qui se détourne partiellement de la vente de rue en raison des craintes concernant la qualité sanitaire des produits qui y sont vendus. À l'inverse, la vente ambulante et de rue reste le mode d'approvisionnement presque exclusif des citadins défavorisés. C'est pourquoi la politique de formalisation des marchés et de restriction de la vente ambulante et de rue peut être interrogée : quelles conséquences ce modèle de distribution alimentaire au sein de la ville a-t-il sur l'approvisionnement des plus pauvres et des moins mobiles ? Pour qui fait-on la ville ? Pour l'heure, on constate davantage une diversification des lieux de vente qu'un remplacement des uns par les autres. Ainsi, le marché de Hang Da, au cœur de la vieille ville, a été rénové et installé au sous-sol d'une galerie marchande ; mais immédiatement, les rues adjacentes ont été occupées, de façon largement informelle, par une multitude de petits étals et de commerces alimentaires de proximité (Di Gregorio, 2013 ; Geertman, 2010 ; Pulliat, 2013).

\section{La ville comme espace pionnier dans la transition alimentaire}

Les habitudes de consommation permettent la même lecture de la diversification des pratiques. L'examen des données du recensement bisannuel sur les conditions de vie (Vietnam Household Living Standards Survey, VHLSS) montre qu'entre 2002 et 2010, en milieu urbain, la consommation de viande est passée de 21,6 à 25,2 kg par personne et par an, celle des produits de la mer de 14,4 à 16,8 kg - soit une hausse de $17 \%$ dans les deux cas, cependant que la consommation de riz a baissé (GSO, 2010, p. 317-341). La consommation de produits laitiers, presque inexistante avant l'ouverture au commerce international, se répand et bénéficie d'une image valorisée auprès des consommateurs - ils sont jugés importants pour la santé des enfants.

On assiste là au modèle classique de la transition alimentaire (Charvet, 2004 ; Landy, 2009) : on passe d'un régime alimentaire essentiellement fondé sur les céréales à un régime plus diversifié, accordant une place croissante aux produits d'origine animale, ainsi qu'aux produits agro-industriels. La transition alimentaire résulte de l'accroissement du niveau de vie qui permet la consommation de calories plus onéreuses, mais aussi des changements de mode de vie, avec le recours accru à des produits nécessitant peu de préparation. Ainsi, elle induit à la fois un accroissement global de la ration, et des modifications structurelles du régime alimentaire (figure 5). De surcroît, on observe qu'elle débute généralement en ville avant que les nouveaux 
Fig. 4 : Évolution de la consommation des principales denrées au Vietnam, 1987-2010.

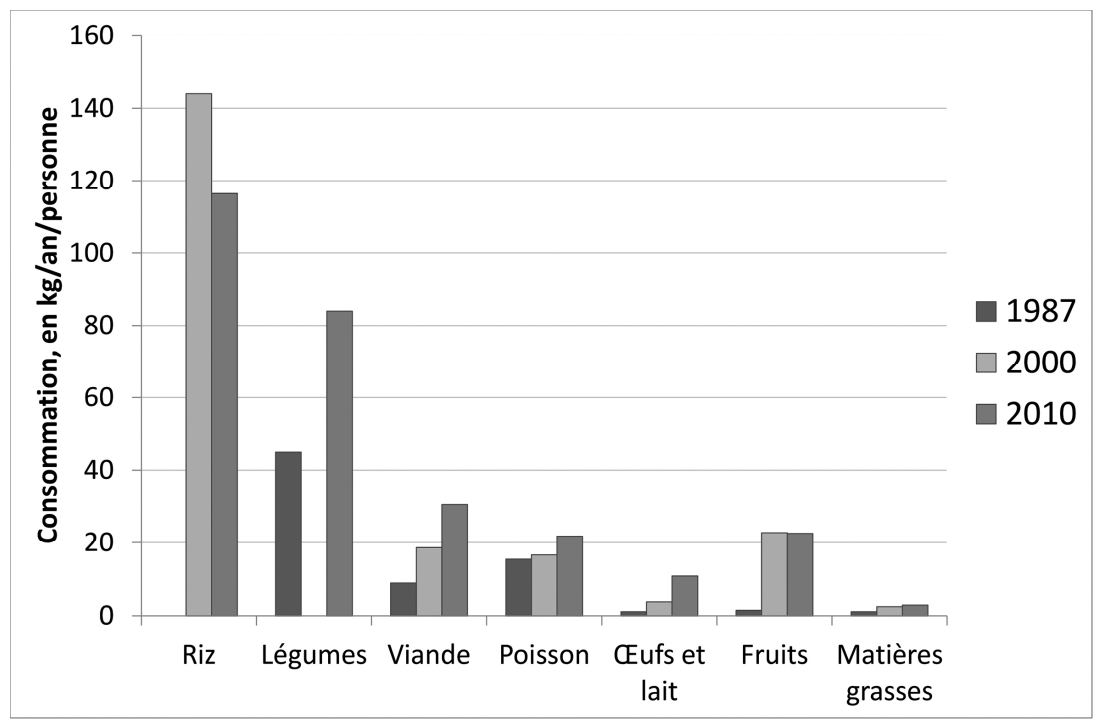

Données : GSO, 2010 ; NIN et Unicef, 2011

modèles de consommation se propagent vers les campagnes environnantes (Landy, 2008 ; Landy, 2009).

Cependant, l'analyse des pratiques alimentaires à l'échelle des ménages donne une image quelque peu contrastée de la transition en cours. Notons d'abord qu'au Vietnam, la part de l'alimentation dans le budget reste élevée à tous les niveaux de revenus, mais la contrainte est sensiblement plus forte pour les plus pauvres. Ainsi, selon les données du VHLSS, en 2010 l'alimentation représentait $62 \%$ des dépenses pour le premier quintile de revenu, contre «seulement » $42 \%$ pour le quintile le plus riche. À titre comparatif, en France, l'alimentation représente $15 \%$ des dépenses des ménages, avec très peu de variations selon les quintiles de revenus (INSEE, 2007). L'effort budgétaire pour l'alimentation reste donc un élément discriminant - et une source de vulnérabilité pour les plus pauvres.

Dans ce cadre, les céréales, et surtout le riz, restent la base du régime alimentaire : elles représentaient en 2005 près de $40 \%$ du volume de l'ensemble des produits consommés contre seulement $15 \%$ pour les produits d'origine animale (Ali, Nguyen The Quan et Ngo Van Nam, 2006). Finalement, tout se passe comme si la structure fondamentale du régime alimentaire restait la même - du riz accompagné de légumes frais, de bouillon, et de plats incluant des produits animaux (mais souvent en petite quantité) -, et que ce sont 
Fig. 5 : Les étapes de la transition alimentaire et leur articulation au budget

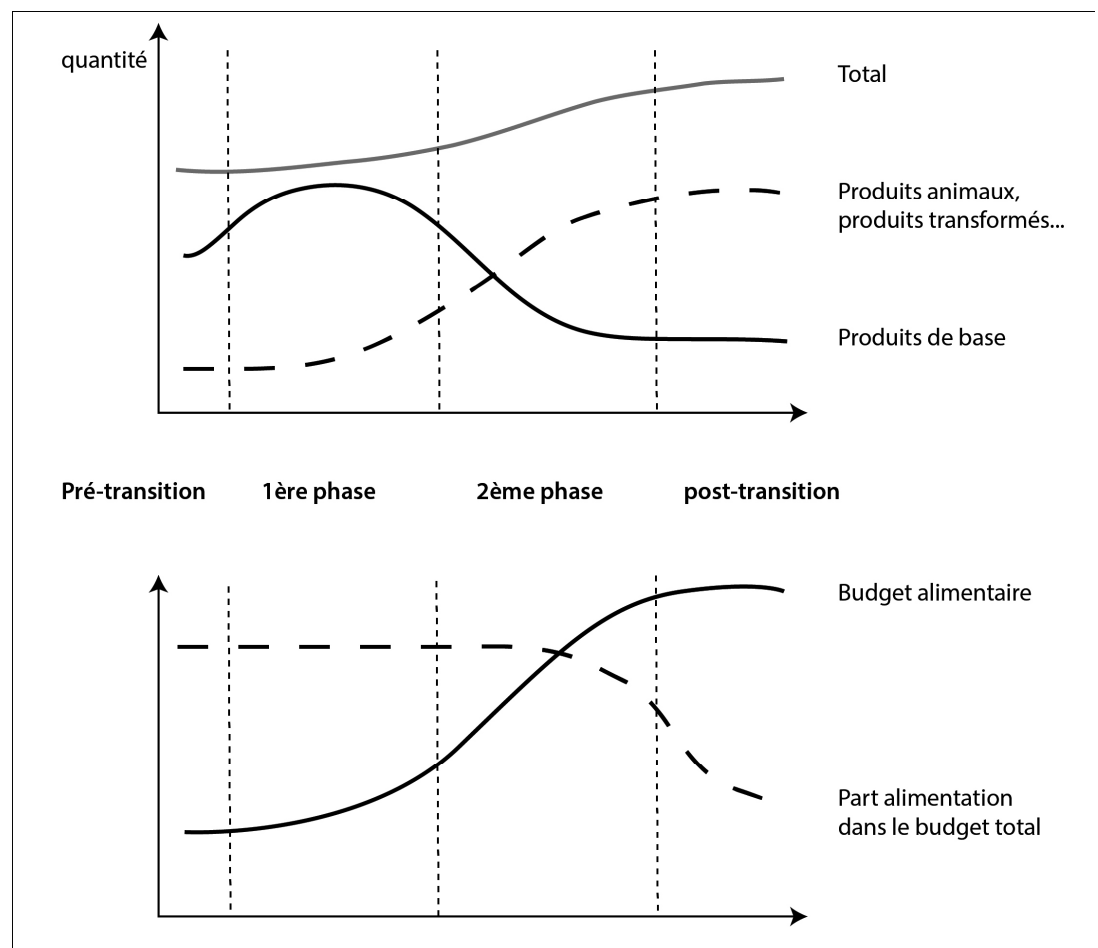

Source : Pulliat, 2013, p. 273

les compléments à ce socle qui, eux, connaissaient une mutation, avec de forts contrastes selon les groupes sociaux. La différenciation sociale se lit dans ces compléments au repas traditionnel. Les aliments valorisés dans le discours des consommateurs, quel que soit leur niveau de vie, sont ceux qui répondent au modèle de la transition alimentaire (produits animaux principalement), voire à une certaine « occidentalisation » (Pingali, 2007) dans le cas des produits laitiers. Or leur consommation croît d'abord en ville et parmi les groupes sociaux favorisés : le changement progressif (mais rapide) des modèles de consommation rend compte d'une certaine polarisation sociale.

\section{Les changements socio-économiques facteurs de transformation des modes de consommation}

Les modes de consommation témoignent également des changements socioéconomiques en ville. À Bangkok, une responsable du programme BioThai (fondation œuvrant pour le développement de l'agriculture biologique) a estimé à près de $70 \%$ la part du budget des ménages consacrée à l'achat de nourriture hors du domicile (repas pris dehors ou achetés tout prêts 
et consommés au domicile) $)^{3}$. Cette donnée nécessite d'être étayée plus précisément mais révèle, quoiqu'il arrive, une tendance très forte évoquée par tous les répondants de l'enquête. Allongement des distances entre domicile et lieu de travail, développement du travail salarié hors du domicile pour les adultes de la famille nucléaire, accroissement des revenus permettant de recourir davantage au marché : autant de facteurs qui favorisent cette transformation alimentaire. Ne pas préparer soi-même (ou un membre du foyer) ses repas, devient un modèle courant, si ce n'est dominant. Cette mutation est cependant rendue possible par un cadre socio-économique particulier : celui où l'emploi informel dans le petit commerce alimentaire reste un débouché économique important pour une main-d'œuvre précaire nombreuse ${ }^{4}$ (Yasmeen et Nirathron, 2014) - notamment migrante (venue des espaces ruraux ou des pays voisins) - produisant un marché alimentaire pléthorique, bon marché et disponible à toute heure. Ce marché se structure en partie en fonction de ces mutations économiques et spatiales : on voit ainsi apparaître de multiples points de vente à proximité immédiate des stations de transport en commun ou des parkings aux heures de pointe. La mobilité de la main-d'œuvre et le fonctionnement du marché alimentaire - en particulier informel - interagissent finement.

\section{L'état nutritionnel : une résultante des évolutions en cours dans les métropoles émergentes}

Ces mutations dans les pratiques alimentaires ont des conséquences dans le statut nutritionnel des consommateurs. Une caractéristique forte des pays émergents est la coexistence de problèmes persistants liés aux carences nutritionnelles et de problèmes nouveaux liés aux excès alimentaires. Le Vietnam a connu une nette amélioration de l'état nutritionnel de sa population. L'insuffisance pondérale, définie par un indice de masse corporelle (IMC) inférieur à 18,5 et qui rend compte d'un déficit énergétique chronique, a connu un déclin marqué : elle concernait 17,2\% des adultes en 2010 contre $38 \%$ en 1990 (NIN et Unicef, 2012). Le taux actuel reste toutefois élevé, et ce même dans les grandes villes : ainsi, une étude portant spécifiquement sur Hô Chi Minh Ville y a évalué la prévalence du sous-poids à 20,4 \% en 2006 (Cuong et al., 2006). À titre de comparaison, selon cette étude, dès 1992, le sous-poids chez les adultes en Chine était de seulement $9 \%$.

Parallèlement, le surpoids et l'obésité connaissent une croissance rapide. Le taux reste bas, puisque cela ne concerne que 5,6\% des adultes à l'échelle

\footnotetext{
3. Entretien, 3 novembre 2016.

4. "Policymakers and scholars need to take small-scale food retailing seriously as an important component of economic security of the under- and unemployed" (Yasmeen et Nirathron, 2014).
} 
nationale en 2012 (NIN et Unicef, 2012) ${ }^{5}$. Cependant cette nouvelle question concerne prioritairement les urbains ${ }^{6}$ (7,6\% des citadins concernés en 2006 (Calandre, 2006), deux fois plus que les ruraux à la même date) et de plus en plus les enfants et les adolescents - ce qui prépare à une augmentation du surpoids chez les adultes de demain. Aussi, la crainte de l'obésité se diffuse à Hanoï : parce que c'est un risque nouveau et en croissance rapide (Slovic, 1987), mais aussi en référence au cas chinois ${ }^{7}$.

Le passage d'une situation nutritionnelle dominée par les enjeux liés aux carences à celle où dominent les problèmes liés aux excès correspond à la « transition nutritionnelle» (Bricas et Seck, 2004 ; Khan et Khoi, 2008 ; Maire et Delpeuch, 2001). Le phénomène n'est qu'amorcé au Vietnam, mais il est rapide, en particulier dans les métropoles. Ce changement dans l'état sanitaire des consommateurs est un marqueur fort des transformations socioéconomiques à l'œuvre dans les métropoles émergentes.

Finalement, l'analyse des transformations des pratiques alimentaires en ville, de l'acquisition des denrées à la situation nutritionnelle qui en résulte, témoigne de l'émergence métropolitaine. L'émergence est ici comprise dans un triple sens :

- d'abord en référence à ses moteurs : le décollage économique lié à l'ouverture internationale, qui induit une forte attractivité pour les investisseurs et les grands groupes internationaux (Jaffrelot, 2008) ;

- ensuite dans son expression sociale : accroissement des disparités entre les espaces et les groupes sociaux, à toutes les échelles d'analyse (Landy, 2007 ; Landy, 2008) ;

- enfin dans sa manifestation socio-économique : développement d'une classe moyenne allant de pair avec l'essor d'une société de consommation (Fleury et Houssay-Holzschuch, 2012).

L'alimentation se révèle alors être une grille efficace d'analyse des dynamiques économiques et sociales liées à l'émergence.

\footnotetext{
5. L’Organisation Mondiale de la Santé définit le surpoids par un IMC supérieur à 25, et supérieur à 30 pour l'obésité, pour les adultes. Mais certaines études définissent un seuil spécifique à l'Asie : le surpoids correspond alors à un IMC supérieur à 23 et l'obésité à un IMC supérieur à 26,1 pour les femmes et 27,3 pour les hommes (Cuong et al., 2006). L'utilisation de ce seuil asiatique induit alors des taux de surpoids supérieurs à ceux évoqués ici : dans l'étude portant sur Hô Chi Minh Ville, cela fait passer le taux de surpoids de $15,4 \%$ à $26,2 \%$.

6. Il serait nécessaire d'étudier quelle part de la prévalence supérieure en milieu urbain est strictement due à des revenus plus élevés qu'ailleurs ; toutefois, on fait l'hypothèse que, toute chose égale par ailleurs, la prévalence en milieu urbain reste plus importante qu'en milieu rural en raison de modèles de consommation différents et d'un mode de vie davantage sédentaire.

7. En Chine, en 2006, en milieu urbain, $17 \%$ des garçons et $10 \%$ des filles de 7 à 18 ans sont touchés par le surpoids et l'obésité (Wu, 2006).
} 


\section{- Alimentation et développement urbain : quelles formes de la ville en devenir ?}

\section{Une perception accrue du risque sanitaire}

Dans le champ alimentaire, le phénomène d'émergence peut être caractérisé par un glissement des craintes des consommateurs de l'insécurité alimentaire (food security) vers l'insécurité sanitaire des aliments (food safety). Évidemment, les enjeux sanitaires ne sont pas absents des espaces les plus touchés par l'insécurité alimentaire, en tant que privation d'accès à une nourriture suffisante tant en quantité qu'en qualité nutritionnelle ; mais dans ce contexte, ils passent au second plan. À l'inverse, l'amélioration des conditions de vie des citadins des métropoles émergentes conduit à un effacement de ces craintes-là au profit de peurs portant sur la qualité des aliments, et surtout sur leur risque toxicologique.

Dans le domaine alimentaire, le risque sanitaire recouvre en effet des sens différents. Les deux principaux sont les suivants :

- le risque biologique, induisant une intoxication alimentaire, lié à une contamination bactérienne des aliments ou de l'eau ;

- le risque toxicologique, qui concerne les résidus de produits phytosanitaires utilisés pour la production, ou éventuellement la conservation des denrées, surtout quand ces produits sont utilisés de façon inadéquate.

À Hanoï les autorités publiques prennent d'abord en compte le risque bactériologique, en développant des normes de stockage, de transport et de distribution des aliments. Par ailleurs, les associations parapubliques locales, notamment l'Union des Femmes, proposent des formations de base aux vendeurs de rue pour promouvoir une meilleure hygiène lors de la préparation ou de la vente de produits alimentaires. Au-delà de l'enjeu de santé publique, cette politique s'inscrit, comme la rénovation des lieux de vente évoquée plus haut, dans la volonté de promouvoir l'image d'une ville « moderne », défaite de l'insalubrité associée au risque sanitaire.

Du côté des consommateurs, les entretiens réalisés ont montré l'importance de la préoccupation sanitaire, puisque plus du quart des répondants l'ont spontanément évoquée. Elle porte sur les deux aspects du risque sanitaire. Dans le premier cas, les répondants évoquaient l'importance de la fraîcheur des aliments pour en assurer la qualité - ce qui explique l' habitude persistante de faire les courses tous les jours (Geertman, 2011 ; Moustier et Dao, 2006). Dans le second cas, c'est l'intensification de l'agriculture et de l'élevage qui engendre l'idée d'une toxicité croissante des aliments (Figuié et al., 2004). C'est une question majeure au Vietnam aujourd'hui, relayée tant par des 
campagnes d'affichage soulignant l'importance de bien laver les produits frais pour en ôter les résidus de produits phytosanitaires, que par les médias. Mais la qualité de l'environnement est aussi évoquée par les répondants. Ainsi, à An Khanh, le processus d'urbanisation à l'œuvre engendre une méfiance même vis-à-vis des produits locaux. L'arrivée d'industries sur le territoire de la commune s'accompagne d'une méfiance concernant les pollutions qu'elles pourraient générer : notamment quant à la qualité de l'eau et aux déchets industriels qui seraient rejetés sans traitement préalable.

\section{Vers une relocalisation de la production alimentaire, pour qui ?}

Ainsi, le développement urbain s'accompagne-t-il d'un processus de « distanciation » (Bricas et Seck, 2004) - par l'accroissement des distances entre zones de production et zone de consommation, par la multiplication des intermédiaires - qui va de pair avec une transformation des risques perçus par les consommateurs urbains.

Toutefois, une part de la défiance des consommateurs vietnamiens est liée au contexte spécifique dans lequel on se trouve. Elle fait notamment écho au manque de confiance général dans les contrôles et les labels, dans un contexte émaillé par des scandales sanitaires récurrents. En Thaïlande à l'inverse, les consommateurs semblent avoir une certaine confiance dans la qualité des produits nationaux (Poupon, 2016). Pour autant, on observe l'apparition d'initiatives militantes en faveur de l'agriculture biologique, diffusant notamment de l'information sur les enjeux sanitaires liés aux produits phytosanitaires. Elles rendent compte de la contamination même des produits vendus comme « biologiques » (BioThai, 2016) et entendent favoriser le redéveloppement d'une agriculture non-marchande, familiale ou communautaire, à proximité des lieux de consommation. C'est ce que fait par exemple le groupe Heart Core Organic, associant système de vente directe entre producteurs et consommateurs et formation à l'agriculture urbaine pour la consommation familiale. Ces initiatives, récentes et très limitées tant en termes de participants que de volumes de produits échangés, sont néanmoins soutenues par un organisme public, la Sustainable Agriculture Foundation. Elles s'inscrivent plus largement dans la lignée du food movement, né en Amérique du Nord, qui promeut un militantisme en faveur d'une relocalisation du système alimentaire et d'une amélioration de l'accès à une alimentation de qualité pour tous.

Au-delà du caractère anecdotique de ces initiatives, la question sous-jacente est cruciale : c'est celle des usages de l'espace. Une réponse à la défiance face à l'approvisionnement traditionnel se trouve en effet dans le recours à la production locale, soit autonome (dans les jardins, sur les espaces 
Fig. 6 : Jardin privé transformé en centre de formation à l'agriculture urbaine biologique à Bangkok, associé au groupe Heart Core Organic.

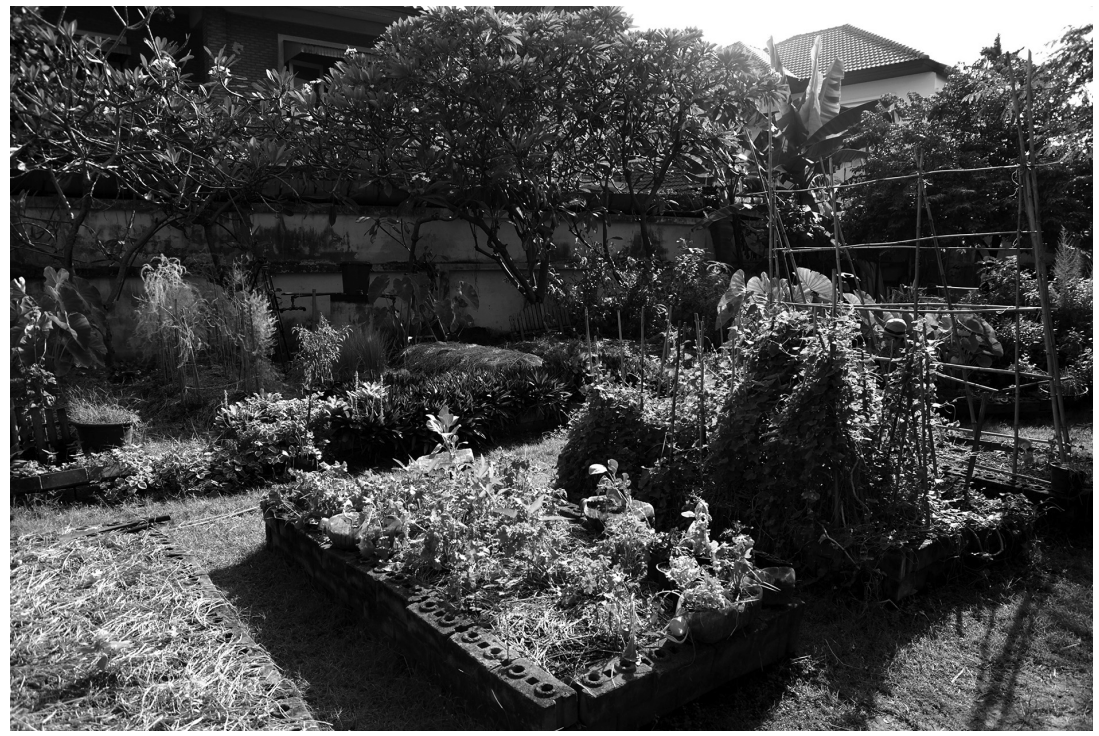

Photo de l'auteur, 2016.

interstitiels, sur les terres agricoles restantes), soit par le développement de filières alternatives où les consommateurs connaissent mieux les producteurs et les lieux de production - ils associent alors cette connaissance à la qualité du produit (Figuié et Mayer, 2010 ; Wertheim-Heck, Vellema et Spaargaren, 2015). Cette question de la sécurité sanitaire se trouve alors être un facteur de résistance et de redéploiement des espaces de production agricole au sein des métropoles ; l'agriculture urbaine bénéficie aujourd'hui, pour ces raisons, d'un certain engouement (Duvernoy et al., 2005 ; Hamilton et al., 2014 ; Tornaghi, 2014), tant au Nord qu'au Sud.

Dans les métropoles émergentes, le phénomène procède de plusieurs dynamiques. D'abord, une dynamique informelle et individuelle conduit, à Hanoï, à la mise en culture de tous les espaces disponibles : les jardins évidemment, mais aussi les berges du fleuve Rouge à Phuc Tan par exemple, comme les espaces non construits dans les périphéries de la ville, ou certaines terres agricoles expropriées mais non encore urbanisées à An Khanh. Il s'agit là d'une forme d'agriculture dans la ville correspondant à une pratique ancienne, rurale. L'enquête menée a montré qu'elle était une ressource alimentaire importante pour les foyers vulnérables (Pulliat, 2015), mais elle concerne également des groupes sociaux favorisés. 
Ensuite, les initiatives militantes évoquées ci-dessus associent la qualité sanitaire de l'alimentation à celle de l'environnement urbain. Un projet financé, entre autres, par l'ONG Oxfam à Bangkok a pour objectif de favoriser le développement des espaces verts ouverts au public, peu nombreux dans la ville-centre. Le projet se fonde sur une réflexion politique sur l'usage des espaces vacants et utilise pour cela le levier alimentaire. En utilisant les espaces vacants comme zone de production agricole, le projet Root Garden entend mobiliser les résidents par cette question alimentaire pour les associer à la réflexion sur les usages de l'espace métropolitain. L'alimentation sert ici de levier en faveur d'une réforme foncière.

Toutefois, cette production de qualité, comme la mobilisation pour bénéficier de l'espace, semble concerner surtout des groupes sociaux globalement favorisés, et surtout très éduqués - même si ces résultats préliminaires restent à étayer. Ces phénomènes de sélection sociale ont été observés et analysés dans les villes du Nord, en particulier en Amérique du Nord, où la différenciation dans l'accès aux espaces d'agriculture urbaine selon le genre et la race a été mise en évidence (Reynolds et Cohen, 2016). Une attention particulière doit donc être portée à ces dynamiques sociales sous-jacentes.

Enfin, les craintes sanitaires ouvrent la voie à un marché associant production contrôlée et lieux de vente considérés comme sûrs - principalement les supermarchés (Figuié et Mayer, 2010). Sautier et al. (2012) évoquent ainsi le cas particulier de l'entreprise Huong Can à Hanoï: l'entreprise cultive depuis 2010 des surfaces en propre, et a passé des contrats avec des producteurs de la proche périphérie de Hanoï. La production répond à des normes de production de qualité (label VietGAP) et est vendue à des supermarchés. Ainsi, la contractualisation permet de valoriser dans le long terme des productions répondant à des normes sanitaires strictes et d'assurer un débouché, pour ces produits, à de petits producteurs. Toutefois, cet exemple reste l'exception : dans leur majorité, les petits producteurs restent exclus des filières de ce type. Moustier et Dao (2010) soulignent que les coopératives de « légumes sûrs » regroupent généralement des producteurs avec des capacités de financement et des surfaces agricoles supérieures à la moyenne. Aussi, si ces initiatives assurent une meilleure rentabilité du foncier agricole de l'hinterland (les revenus générés sont supérieurs aux revenus agricoles moyens) tout en répondant à une forte demande de qualité, elles restent inaccessibles à de nombreux petits producteurs pauvres. On peut donc s'interroger sur les dynamiques sociales du développement de ces filières : quels producteurs y ont effectivement accès ? À qui ce marché bénéficie-t-il ? 
Au total, hors les initiatives individuelles et largement informelles, tant du côté des consommateurs que des producteurs, l'agriculture urbaine et périurbaine qui se déploie semble bénéficier d'abord à des groupes sociaux plutôt favorisés, qui disposent de ressources (éducatives, politiques, économiques) pour tirer parti de ces dynamiques alimentaires et pour mobiliser l'espace urbain et périurbain dans ce sens-là. Là encore, ces hypothèses nécessitent d'être davantage examinées

, mais elles conduisent à interroger l'existence d'un processus de gentrification de l'agriculture urbaine et périurbaine. À cet égard, un courant critique commence à interroger ces dynamiques urbaines (Tornaghi, 2014). Dans les métropoles émergentes, il s'agit bien d'une interrogation : les résultats préliminaires suggèrent que les espaces concernés par cette nouvelle agriculture urbaine orientée vers la qualité (et les groupes sociaux favorisés) ne sont pas les mêmes que ceux de l'ancienne production intra et périurbaine menacés par l'urbanisation. En ce sens, il y a davantage une polarisation croissante des dispositifs alimentaires qu'une gentrification de la production urbaine à proprement parler. Quoi qu'il en soit, l'alimentation révèle bien, là encore, les tensions pour les usages de l'espace et les dynamiques sociales à l'œuvre dans les métropoles émergentes.

\section{- Conclusion}

Ouverture internationale et intégration régionale, formalisation des canaux de distribution, différenciation croissante des modèles de consommation : l'entrée alimentaire donne à voir finement les mutations sociales, économiques et spatiales caractéristiques de l'émergence dans les métropoles des Suds. L'empreinte spatiale de l'alimentation (espaces de production, espaces de distribution, espaces de consommation) révèle également les usages et des pratiques de la ville, en rendant compte tout à la fois des tensions foncières, des flux des produits, des mobilités des personnes, des manières d'habiter la ville qui émerge.

En ce sens, l'alimentation révèle la forme de la ville. Mais, au-delà encore, l'alimentation ré-forme la ville: l'importance croissante accordée aux espaces agricoles dans les villes et leurs périphéries, répondant aux préoccupations sanitaires des habitants ou aux politiques environnementales urbaines (gestion de l'eau, de la qualité de l'air, des îlots de chaleur urbains etc.), conduit à repenser l'aménagement des villes, les usages de leurs espaces. 


\section{$\nabla$ Bibliographie}

Ali M., Nguyen The Quan et Ngo Van Nam (2006), «An analysis of food demand patterns in Hanoi: predicting the structural and qualitative changes », Technical Bulletin, n०35, Taiwan, AVRDC.

Anjaria J.S. (2006), «Street hawkers and public space in Mumbai », Economic and Political Weekly, p. 2140-2146.

BioThai (2016), « Thai PAN has published the results of their second round of testing on chemical pesticide residues for 2016 », http://www.biothai.org/node/1427.

Bognon S. (2015), « Nourrir Paris : trajectoire de l'approvisionnement alimentaire de la métropole capitale, de la fin de l'Ancien Régime à nos jours », Géocarrefour, vol. 90, $n^{\circ} 90 / 2$, p. 163-171.

Bricas N. et Seck P.A. (2004), « L'alimentation des villes du Sud : les raisons de craindre et d'espérer », Cahiers Agricultures, vol. 13, n¹, p. 10-14.

Calandre N. (2006), Pratiques et perception des risques nutritionnels : Les mères face aux malnutritions infantiles au Vietnam, Thèse de doctorat, Montpellier, Université Montpellier I.

Carletto C., Zezza A. et Banerjee R. (2013), « Towards better measurement of household food security: Harmonizing indicators and the role of household surveys », Global Food Security, vol. 2, $\mathrm{n}^{\circ} 1$, p. $30-40$.

Charvet J.-P. (2004), L'alimentation dans le monde : mieux nourrir la planète, Paris, Larousse.

Coates J., Swindale A. et Bilinsky P. (2007), « Household Food Insecurity Access Scale (HFIAS) for measurement of food access: indicator guide », Food and Nutrition Technical Assistance Project, Academy for Educational Development.

Cohen M.J. et Garrett J.L. (2010), "The food price crisis and urban food (in) security », Environment and Urbanization, vol. 22, n², p. 467-482.

Courrier du Vietnam (2012), « Les marchés de Hanoi repoussés en banlieue », Le Courrier $d u$ Vietnam, 17 mai 2012.

Cuong T.Q., Dibley M.J., Bowe S., Hanh T.T.M. et Loan T.T.H. (2006), « Obesity in adults: an emerging problem in urban areas of Ho Chi Minh City, Vietnam », European Journal of Clinical Nutrition, vol. 61, n5, p. 673-681.

Dao T.A. et Fanchette S. (2008), « La crise alimentaire mondiale : une opportunité pour relancer la filière rizicole au Vietnam ? », Hérodote, vol. 131, n4, p. 175-194.

Dessus S., Herrera S. et De Hoyos R. (2008), « The impact of food inflation on urban poverty and its monetary cost: some back-of-the-envelope calculations », Agricultural Economics, vol. 39, p. 417-429.

Di Gregorio M. (2013), « Hanoi Public Market », https://www.youtube.com/watch?v=78m8VetEWTU.

Duteurtre G. et Pannier E. (2016), «Local Trajectories in the Vietnamese Transition to Market Economy: Alliances Between Firms, Farmers and Government Officials in the dairy industry », SASE 28th Annual Conference, University of Berkeley California.

Duvernoy I., Jarrige F., Moustier P. et Serrano J. (2005), « Une agriculture multifonctionnelle dans le projet urbain: quelle reconnaissance, quelle gouvernance », Les Cahiers de la multifonctionnalité, vol. 8, p. 87-104. 


\section{Métropoles émergentes et alimentation : une lecture croisée}

Ericksen P.J. (2008), « Conceptualizing food systems for global environmental change research », PDS, vol. 18, n¹, p. 234-245.

Figuié M., Bricas N., Thanh V.P.N. et Truyen N.D. (2004), « Hanoi consumers' point of view regarding food safety risks: An approach in terms of social representation », Vietnam social sciences, vol. 3, n¹01, p. 6372.

Figuié M. et Mayer J. (2010), «A qui se fier quand les aliments font peur? Labels, points de vente et décontamination symbolique au Vietnam », Working Paper, n²010-4, Montpellier, UMR MOISA.

Fleury A. et Houssay-Holzschuch M. (2012), «Pour une géographie sociale des pays émergents », EchoGéo, n²1.

Geertman S. (2010), « Urban development trends in Hanoi \& impact on ways of life, public health and happiness », conférence Hanoi Millennium - City Past and Future, UN-Habitat and Global Research Center Hawaii.

Geertman S. (2011), "Fresh Markets, a way of life and public health under threat », Vietnamese Urban Planning Journal (Tap Chi Xay Dung).

GSO (2010), «Vietnam Household Living Standards Survey », Hanoi, Office Général de la Statistique.

Hamilton A.J., Burry K., Mok H.-F., Barker S.F., Grove J.R. et Williamson V.G. (2014), « Give peas a chance? Urban agriculture in developing countries. A review », Agronomy for Sustainable Development, vol. 34, n¹, p. 45-73.

INSEE (2007), «Enquête Budget de famille », Insee Résultats - Société, n73, Institut National de la Statistique et des Etudes Economiques.

Jaffrelot C. (2008), L'enjeu mondial : les pays émergents, Paris, Les Presses de Sciences Po, $381 \mathrm{p}$.

Janin P. (2009), "Les "émeutes de la faim" : une lecture (géo-politique) du changement (social) », Politique étrangère, vol. Eté, n², p. 251-263.

Khan N.C. et Khoi H.H. (2008), « Double burden of malnutrition: the Vietnamese perspective », Asia Pacific Journal of Clinical Nutrition, vol. 17, nSuppl 1, p. 116-118.

Landy F. (2007), L'Inde ou le grand écart, La Documentation Française coll. "La Documentation Photographique".

Landy F. (2008), « Nourrir 2,5 milliards de personnes, plus et mieux : les défis de l'Inde et la Chine », in Nourrir les hommes, SEDES, pp. 184-224.

Landy F. (2009), « India, "Cultural Density" and the Model of Food Transition », Economic and Political Weekly, pp. 59-61.

Maire B. et Delpeuch F. (2001), « Les risques de la transition alimentaire », La Recherche, n³39, p. 102-104.

Maxwell D., Levin C., Armar-Klemesu M., Ruel M., Morris S. et Ahiadeke C. (2000), Urban livelihoods and food and nutrition security in Greater Accra, Ghana, Washington DC, International Food Policy Research Institute (IFPRI), 172 p.

Monnet J. (2006), « L'ambulantage : Représentations du commerce ambulant ou informel et métropolisation », Cybergeo : European Journal of Geography. 
Moustier P. et Dao T.A. (2006), Supermarkets and the poor in Vietnam, Hanoi, Markets and Agriculture Linkages for Cities in Asia (Malica).

Moustier P. et Dao T.A. (2010), « L'accès des petits producteurs aux filières de qualité au Vietnam. Promouvoir l'information et la coopération. », Perspective, $n^{\circ} 5$, Cirad.

NIN et Unicef (2011), « A review of the nutrition situation in Viet Nam - 2009-2010 », Hanoi, National Institute of Nutrition.

NIN et Unicef (2012), « Summary report - General Nutrition Survey 2009-2010 », Hanoi, National Institute of Nutrition.

Pingali P. (2007), « Westernization of Asian diets and the transformation of food systems: Implications for research and policy », Food Policy, vol. 32, n³, p. 281-298.

Pothukuchi K. et Kaufman J.L. (1999), «Placing the food system on the urban agenda: The role of municipal institutions in food systems planning ", Agriculture and Human Values, vol. $16, \mathrm{n}^{\circ} 2$, p. 213-224.

Poupon R. (2011), «L'agriculture thaïlandaise, un buisson d'alternatives », in Stéphane Dovert et Jacques Ivanoff (dir.), Thaïlande contemporaine, Paris - Bangkok, IRASEC - Les Indes Savantes.

Poupon R. (2016), Les conglomérats agro-industriels sud-est asiatiques, Carnet de l'Irasec, $170 \mathrm{p}$.

Pulliat G. (2013), Vulnérabilité alimentaire et trajectoires de sécurisation des moyens d'existence à Hanoi : une lecture des pratiques quotidiennes dans une métropole émergente, Thèse de doctorat, Nanterre, Paris Ouest - Nanterre, 374 p.

Pulliat G. (2015), « Food securitization and urban agriculture in Hanoi (Vietnam) », Articulo - Journal of Urban Research, $\mathrm{n}^{\circ}$ Special issue 7.

Rastoin J.-L. et Ghersi G. (2010), Le système alimentaire mondial: Concepts et méthodes, analyses et dynamiques, Editions Quae, $586 \mathrm{p}$.

Reynolds K. et Cohen N. (2016), Beyond the Kale: Urban Agriculture and Social Justice Activism in New York City, University of Georgia Press.

Sautier D., Dao T.A., Pham C.N. et Nguyen N.M. (2012), « Agriculture et croissance urbaine à Hanoi », 9ème forum économique et financier franco-vietnamien : Pour une urbanisation durable de la périphérie des villes au Vietnam.

Sen A. (1981), Poverty and Famines: An Essay on Entitlement and Deprivation, 0xford University Press, 276 p.

Sobal J., Kettel Khan L. et Bisogni C. (1998), « A conceptual model of the food and nutrition system », Social Science \& Medicine, vol. 47, n7, p. 853-863.

Tornaghi C. (2014), « Critical geography of urban agriculture », Progress in Human Geography, vol. 38, n4, p. 551-567.

Wertheim-Heck S.C.0., Vellema S. et Spaargaren G. (2015), « Food safety and urban food markets in Vietnam: The need for flexible and customized retail modernization policies », Food Policy, vol. 54, p. 95-106.

Wu Y. (2006), "Overweight and obesity in China », BMJ : British Medical Journal, vol. 333, $\mathrm{n}^{\circ} 7564$, p. 362-363. 
Métropoles émergentes et alimentation : une lecture croisée

Yasmeen G. et Nirathron N. (2014), « Vending in Public Space: The Case of Bangkok », WIEGO Policy Brief (Urban Policies), vol. 16, p. 1-18. 